\title{
Protée
}

\section{" Le théâtre de ma mort ". La création comme annihilation}

\section{Dafna Ben-Shaul}

Volume 27, numéro 1, 1999

La Mort de Molière et des autres

URI : https://id.erudit.org/iderudit/030548ar

DOI : https://doi.org/10.7202/030548ar

Aller au sommaire du numéro

Éditeur(s)

Département des arts et lettres - Université du Québec à Chicoutimi

ISSN

0300-3523 (imprimé)

1708-2307 (numérique)

Découvrir la revue

Citer cet article

Ben-Shaul, D. (1999). « Le théâtre de ma mort ». La création comme annihilation. Protée, 27(1), 93-98. https://doi.org/10.7202/030548ar

\section{Résumé de l'article}

Cet essai s’arrête sur trois dénominateurs sémantiques communs établis dans l'oeuvre de Wilson par des liens associatifs entre des images visuelles, verbales et tonales: la mort, l'hérésie et l'absence. Ils sont liés, par le thème de la création, au créateur et, plus spécifiquement, à Molière. Le créateur mourant, dévoré par sa création, est représenté dans La Mort de Molière à la fois comme un processus et son produit - le sujet observé, «un mourant au travail». La mort devient ainsi une action constitutive. Mais Molière est aussi un hérétique, un Dom Juan, un illusionniste, qui, par le fait de créer par la négation, va jusqu'à défier le Créateur. Mais un créateur est aussi celui dont l'oeuvre est produite et conservée même en son absence. Cette absence est l'objet d'une représentation, elle devient l'objet d'un fétichisme. Ces aspects permettent une interprétation de La Mort de Molière comme présentation de la création comme annihilation. 


\section{«LE THÉÂTRE DE MA MORT» LA CRÉATION COMME ANNIHILATION}

Traduit de I'anglais par Jean-Pierre Vidal

DAFNA BEN-SHAUL

\section{L'INTERPRÉTATION ASSOCIATIVE}

Dans une interview avec Umberto Eco, Wilson décrit l'expérience du spectateur comme un acte d'association:

On peut faire des associations libres entre toutes les parties de l'oeurre et de bien des façons. Il n'y a pas de façon bien définie de lier les diverses images, les divers sons et les autres unités d'information. Il existe au contraire un nombre infini de possibilités: l'œuvre est un espace dans lequel nous entendons, nous voyons et nous vivons une expérience; et ensuite nous faisons des associations. ${ }^{1}$

Malgré l'objection non dépourvue d'ambiguité que lui fait ici Wilson, Eco rattache cette déclaration au concept d'interprétation. Il tempère également la tendance qu'a Wilson à refuser de faire du créateur un partenaire impliqué dans ce processus d'interprétation-création, en prétendant que l'idéologie et la mise en scène orientée vers l'interprétation n'exigent pas nécessairement une direction ferme et dépourvue d'ambiguité. On peut au contraire reconnaître une invite à l'interprétation dans les accents placés dans l'œuvre par le biais du choix des matériaux, de la forme et du rythme, qui tous influencent les associations faites par le spectateur. Il apparaît, si l'on adopte la position d'Eco, que dans le travail de Wilson la frontière entre traits de signification et traits affectifs (déjà mince au départ) se trouve brouillée, au point qu'on peut les percevoir comme complémentaires.

Cette possibilité de reconnaître que le langage de Wilson est une invite à l'interprétation est également évoquée par l'observation méthodologique suivante de Christopher Innes, dans son Avant Garde Theatre:

La structure [des pièces de Wilson] est musicale, en ce sens que l'action s'y résume à une combinaison architecturale de sons, de mots et de mouvements, dans laquelle les images sont répétées telles quelles ou modifiées de manière à former des motifs thématiques. ${ }^{2}$

Il est possible d'associer les images qui apparaissent dans l'œuvre à des connotations dans l'esprit du spectateur. On peut ainsi comprendre le concept de «motif thématique» comme un dénominateur commun sémantique appliqué - au moins par le spectateur - aux images visuelles, verbales et sonores de l'œuvre. 
L'acte d'association se fonde aussi sur la possibilité de reconnaître un lien entre les images elles-mêmes et d'autres éléments, tels que les textes théoriques, biographiques ou dramatiques. De ce point de vue, l'association est une procédure intertextuelle. Les dénominateurs communs sémantiques peuvent être interprétés, littéralement ou métaphoriquement, comme des aspects de la signification et peuvent ainsi se fondre l'un dans l'autre. Malgré la capacité ou même la tendance qu'a l'interprétation associative de devenir un processus intégrateur, rien n'autorise à considérer l'une ou l'autre des couches interprétatives qu'elle rend possibles comme le thème central ou le sens général de l'œuvre.

Pour bien montrer la formation d'une de ces couches interprétatives dans La Mort de Molière, je m'attacherai essentiellement à l'idée de mort et, à un moindre degré, aux idées d'hérésie et de vacuité (ou de vide). Leur présence explicite en tant qu'images (l'agonie, les allusions à Dom Juan, un fauteuil vide, etc.), rend perceptible un processus associatif plus indirect et plus complexe. Fondamentalement, la mort, l'hérésie et la vacuité se trouvent rattachées de façon elliptique à l'acte de création en général et pas seulement à la création moliéresque. Ainsi peut se construire pour l'œuvre de Wilson une interprétation intégratrice, pour laquelle la création se présente comme un acte d'annihilation ou d'évidement.

\section{LA MORT}

À propos de la relation entre l'art et la psychologie, Jung décrit un type d'artiste qui se consume dans l'accomplissement de sa destinée. Dans cette situation, l'artiste n'est plus un individu mais le médium de sa création ${ }^{3}$. Dans Le Roman de Monsieur de Molière, Boulgakov (qui d'après Jan Linders fut une des sources d'inspiration de La Mort de Molière) semble faire écho à cette observation. Il décrit la vie de Molière comme l'émergence d'une énergie créatrice dont les effets s'inscrivent sur le corps du sujet, à commencer par la faiblesse de Molière à sa naissance (dans un dialogue qui unit à travers le temps le narrateur et la sage-femme) et sa description comme un enfant maladif. Les effets des misères que sa troupe et lui subissent sont décrits de façon récurrente. Par exemple, dans le contexte de la critique déchaînée contre L'École des femmes:

Mon héros en sortit malade - une toux suspecte s'était déclarée

-, fatigué et animé d'un étrange état d'esprit; ce n'est que par la suite que l'on attacha à cet état la très sérieuse appellation médicale d'hypocondrie. 4

Ainsi la frontière qui sépare la dégénérescence physique et mentale du créateur, en l'occurrence Molière, de l'acte de création se trouve constamment obscurcie jusqu'à ce que le sujet et l'objet de sa création - l'invalide réel et imaginaire - finissent par se fondre, symboliquement et ironiquement, d'une façon «tragi-comique».

La première image visuelle de La Mort de Molière est un portrait promené par une jeune fille (Isabelle); peint par Mignard, il représente Molière sous les traits du César de La Mort de Pompée de Corneille. Immédiatement après, on entend le poème qui parle de Molière sans en parler vraiment:

Molière c'est la naissance de la comédie venant de l'esprit

De dégoût Molière c'est une danse

Sur une petite scène parmi les poignards affûtés du clergé (section 1)

La première apparition du «Roi de la comédie» sous les traits du «César de la tragédie», alors qu'on l'identifie verbalement à la naissance de la comédie (acte d'annihilation), et la description de sa danse macabre entre les couteaux ne font pas référence à sa mort en février 1673. Et les mots "Molière se meurt", avec toute la durée de ce présent qui constitue un thème verbal, ne fonctionnent pas non plus comme un simple renforcement des images visuelles de l'agonie. La mort comme durée, renforcée par la pulsation de la musique de Glass, inclut apparemment le Molière vivant dont l'acte créateur a besoin de la proximité de la mort et se trouve même décrit métaphoriquement comme la mort ou l'auto-annihilation.

Le fait que Molière soit «un mourant au travail» (section 1) trouve son expression concrète dans la 
présence de ceux qui pourraient être vus comme des témoins à son chevet, témoins de sa vie, de sa mort et de son accomplissement artistique, témoins dont la présence déploie toute la durée d'une vie. Ce sont, d'abord représentées par leurs yeux, les femmes de sa vie, y compris Madeleine pourtant déjà morte à l'époque (puisqu'elle est morte en 1672), et un homme à moustaches qui pourrait être La Grange, Baron ou Chapelle ${ }^{5}$. L'image de ces témoins transforme l'agonie en performance, dans laquelle le sujet-créateur, le processus de création et l'objet se trouvent réunis. La proximité entre la deuxième séquence verbale (qui décrit Molière s'obstinant à vouloir jouer Le Malade imaginaire) et celle qui la suit, commençant par «le monde entier est une scène», avec la répétition de la phrase "Molière se meurt" (section 3), suivies du retour au leitmotiv visuel de Molière à l'agonie dans son lit, permet d'associer le malade ou sa maladie à l'engagement constant et mortel dans l'imaginaire ${ }^{6}$. La métaphore du theatrum mundi ne décrit pas seulement la vie comme un théâtre, mais assimile également le jeu théâtral à la consomption organique du créateur, ici Molière.

Le texte d'Hemingway (section 10 de la version allemande) met en scène des soldats blessés qui gisent immobiles, incapables de manifester qu'ils sont encore vivants, ne serait-ce qu'en s'asseyant, jusqu'à ce que finalement les oiseaux qui les regardent - le corbeau noir et blanc est du nombre - commencent à picorer leurs corps (y compris leurs yeux). On peut alors voir Molière s'asseyant dans son lit, puis le rituel des religieuses qui s'affairent autour du lit blanc, avec force mouvements de bras et en poussant un cri. Bien que les religieuses soient liées au motif du témoin, leur présence est bien plus activement et agressivement associée aux becs des oiseaux qui observent d'en haut et aux couteaux ouverts du clergé. L'impression que le créateur dépend du regard qui le transforme en objet s'en trouve renforcée. Le regard est la cause de cette volonté de «s'asseoir» ou, en alternance, de continuer à danser «sur une petite scène». Le regard n'est pas seulement extérieur, il est aussi introspectif et la prise de vue en plongée rappelle les descriptions, faites par les personnes qui en ont fait l'expérience, de ce moment où le mourant se voit lui-même. Sous cet angle, on peut voir "Moi Ma mort» (section 10) et le monde comme un "paysage de Ma mort» ou comme «Le théâtre de ma mort» (section 9). Se juxtaposent à ces phrases la destruction des manuscrits par le feu et les doigts s'ouvrant sur le papier brûlé qui reçoit ainsi l'empreinte de la main. De cette façon, le papier brûlé peut être vu comme un miroir de la main ou comme une peau. Cette action unit le créateur à sa création par l'accomplissement d'un acte d'annihilation qui est aussi une mort métaphorique.

À la perception de l'image de Molière ainsi construite, il importe d'ajouter la couche métalinguistique selon laquelle, comme le dit le texte de Müller qui accompagne la vidéo, «Le poème observe un mourant au travail» (section 1). Boulgakov prétend que même si les manuscrits de Molière ont été brûlés, ses œuvres n'en sont pas moins devenues des joyaux de la culture éternelle. De son point de vue rétrospectif, l'association de la mort organique à l'éternité de la création ne fait que souligner davantage le statut de "roi du théâtre français» reconnu à Molière: Molière est vraiment immortel ${ }^{7}$. Mais cette survie sans âge veut-elle dire que Molière est immortel ou plutôt que sa transformation en icône est le signe d'un autre genre de mort?

Il est fort possible que l' «énigme" que pose l'œuvre de Wilson quant au véritable objet de sa description (Wilson en Molière? Molière en Müller?) ne soit pas aussi signifiante que la naissance de la création à partir d'un acte d'annihilation: «Ce n'est pas un poème sur Molière» (section 1). On pourrait ajouter à cela le portrait encadré représentant le créateur sous les traits d'un des personnages qu'il a joués (faisant ainsi peut-être écho à la décision de Wilson de s'impliquer dans la mort de Molière après avoir vu le tableau représentant cette mort ${ }^{8}$. "Je ne suis pas Hamlet", dit l'acteur qui joue Hamlet dans Hamlet Machine, et vers la fin de cette phrase on voit la photo de l'auteur (Müller) se faire mettre en pièces ${ }^{9}$. De même que l'on n'est pas le personnage qu'on joue, on 
n'est pas non plus le «moi» peint ou photographié. La quête de l'identité ou du moi profond s'exprime dans la création de personnages et la production d'autoreprésentations fondées sur la négation de l'existence d'un sujet. Mais ces créations ne font qu'ouvrir la voie à leur tour à leur propre annihilation, au profit d'autres objets. Au lieu de construire un récit fiable de la vie de Molière vue à travers le prisme de sa mort, Wilson braque un objectif kaléidoscopique sur cette vie en assimilant le sujet à un nouvel objet cadré.

Une collection de fragments se réorganise et devient un collage par l'action du montage, ce montage dont Pasolini disait:

[...] aussitôt que le montage intervient [...] le présent devient le passé [...]. C'est par la mort que nos vies deviennent expressives. Le montage accomplit ainsi pour la matière filmique [...] ce que la mort accomplit pour la vie. 10

On pourrait ajouter à l'assimilation métaphorique de la mort au montage les mots de Roland Barthes sur la photographie:

[...] lorsque je me découvre sur le produit de cette opération [la photographie], ce que je vois, c'est que je suis devenu ToutImage, c'est-à-dire la Mort en personne. ${ }^{11}$

La création rétrospective, qui transforme Molière et sa mort en objet, se trouve réfléchie dans la présence implicite du médium cinématographique qui se manifeste par la profusion des allusions visuelles au cadre ou au plan, comme la "scène» blanche, les carreaux du plancher, les châssis des fenêtres et leurs projections lumineuses. Il s'agit d'images blanches (vierges) qui permettent, comme le travail de montage de Wilson et Müller, des jeux de combinaison qui n'ont rien à voir avec l'ordre biographique. Le télescope de Galilée peut lui aussi être perçu comme une allusion à l'appareillage cinématographique. Le regard du savant est médiatisé par les lentilles, extensions externes de la rétine (que l'on voit immédiatement avant, dans l'image des trois paires d'yeux). Tournant son télescope vers l'indifférence des étoiles, Galilée illustre le fossé qui sépare celui qui regarde de l'objet de son regard. Cette image crée une analogie ironique entre l'institution scientifique et l'institution artistique-cinématographique. Ainsi des phrases comme «Molière n'est pas un sujet de poésie/ Molière c'est un objet pour la médecine» (section 1) peuvent-elles être interprétées comme de l'auto-ironie. La transformation de Molière en objet trouve une autre manifestation frappante dans l'image du médecin moderne. Le médecin a un contact non médiatisé avec l'urine, mais il n'a pas d'accès réel à son patient, et il se trouve identifié au point de vue rétrospectif non seulement parce qu'il appartient à notre époque, mais aussi parce qu'il réapparaît après le générique final.

\section{L'HÉRÉSIE}

Comme la mort, l'hérésie est un acte d'annihilation associé à la création. Un lien associatif clair est établi entre Molière et l'idée d'hérésie par la description que fait Sganarelle de Dom Juan (section 3), qu'il présente comme un démon, une bête, un épicurien et un hérétique, tandis qu'apparaît le renard. Le renard, symbole médiéval de forces sataniques, est d'abord vu comme une représentation obscure de l'élément démoniaque et animal mentionné par le texte, souligné par le son du claquement d'un fouet. Puis il apparaît avec un corps d'homme, comme le permet sa fonction allégorique, semblable à celle qu'il occupe dans les fables de La Fontaine, et son assimilation au personnage du trickster. Le renard regarde vers l'avant, en une sorte de collusion provisoire avec le spectateur hors champ; puis, entre les rideaux blancs, il contemple Molière gisant dans son lit. Ainsi il conjoint les figures de Dom Juan, du trickster démoniaque et de Sganarelle. Ce dernier observe les actions de Molière/Dom Juan en manifestant son attachement à l'ordre établi, mais aussi en complice de ses machinations.

Dans un tel contexte, on peut assimiler Molière au trickster démoniaque et masqué qui peut lui-même devenir une victime ${ }^{12}$. Le fait que le renard observe Molière peut être interprété de diverses façons qui impliquent, comme la position de Sganarelle, à la fois 
le rejet de l'hérésie et le recours à l'alternative carnavalesque perturbatrice qu'il propose. Ainsi l'hérésie est-elle non seulement religieuse et sociale, mais elle se trouve en outre assimilée à la figure du créateur, en particulier le créateur comique. L'acte de création, cependant, n'est pas nihiliste, il offre une vérité alternative. C'est un acte de liberté qu'exprime aussi le sens étymologique du mot hérésie, puisque hairesis veut dire "choix» en grec. Galilée, dont les découvertes n'auraient pas été possibles n'eût été de son «hérésie», est aussi en rapport avec ce thème. La création semble faire concurrence aux vérités cosmiques ou même à la Création elle-même. Ainsi le créateur/Molière se trouve-t-il placé entre la sphère démoniaque et la sphère céleste, entre le blanc et le noir (ceux, par exemple, des figures de la commedia dell'arte), «Entre ange et bête» (intertitre de la section 1).

Le lien entre les deux types d'annihilation - la mort et l'hérésie - est établi très clairement par l'image du feu. Le feu de l'enfer qui attend l'hérétique est aussi le feu de sa vie, une force dionysiaque dont la nature dévastatrice peut être assimilée, dans l'œuvre de Wilson, à l'acte de création.

\section{LA VACUITÉ}

Dans l'œuvre de Wilson, l'annihilation se manifeste non seulement par les images de mort et d'hérésie, mais aussi par l'acte qui consiste à réduire l'existence à un rien, physiquement et mentalement, par la vacuité. La vacuité n'attribue pas l'acte de création à la présence de la mort ou du créateur démoniaque, mais à leur absence. Le fauteuil vide est non seulement une métonymie de Molière, mais aussi une métaphore de la création comme annihilation. En un sens, le fauteuil placé dans le carré blanc peut lui aussi être perçu comme une scène. Il sert de support à tout le potentiel d'images qu'éveillent la mémoire ou le théâtre. Comme l'imagination telle que la décrit Sartre, l'existence du monde y semble niée, au profit de la fraîcheur et de l'autonomie de l'image qui surgit dans la conscience ${ }^{13}$. La séquence du clavecin avec la femme et l'enfant peut, elle aussi, être perçue de cette façon. Par une sorte de miracle qui rattache peut-être aussi la création moliéresque à l'esprit poétique de sa mère -, on voit l'instrument jouer tout seul, ne nous laissant apparemment que la musique, alors qu'en fait celle-ci est produite par un interprète invisible ${ }^{14}$.

Quoi qu'il en soit, l'image du fauteuil pris dans une cage de verre non seulement fait apparaitre le sujet, mais l'assimile à l'objet en les rattachant tous deux à la vacuité, au vide. Elle offre non seulement une représentation de Molière mais un objet de musée, presque un fétiche. Comme la médiation de l'objectif de la caméra, la cage de verre nous sépare de l'objet. Le bruit du verre qui se brise demeure une option non réalisée dont la présence se limite à la bande-son. Ce bruit se rattache également à l'apparition, derrière la vitre de la cage, du médecin moderne dont l'accès à son patient reste bloqué et médiatisé.

La mort, l'hérésie et la vacuité décrivent donc l'acte de création comme un acte d'annihilation essentiellement l'annihilation du sujet mais aussi la négation de la réalité de l'objet. Tous ces éléments se trouvent reliés de bien des façons: par exemple, lorsqu'on voit le père de Molière - qui peut être interprété comme une représentation de Molière luimême - en train de cirer le cuir du fauteuil et que l'on entend la phrase «Father, I am dying» (section 5). La signification de cette phrase est multiple: le besoin paternel d'assurer sa descendance dans la pratique de la tapisserie implique un arrêt de mort pour Molière, mais la mort peut aussi être interprétée comme la destruction et la vacuité liées à l'homme-théâtre qu'est Molière, et l'action du père peut alors être interprétée comme une tentative désespérée de les ramener à la vie. Le feu qui dévore les chaises représente un pouvoir à la fois destructeur et créateur, qui connote non seulement l'hérésie mais renvoie, en outre, au statut d'inexistence attribué à la fois au sujet et à l'objet. Le lien entre la mort, l'hérésie et la vacuité est à nouveau visible dans la scène du tombeau où Molière descend dans des flammes semblables à celles qui engloutissent Dom Juan, non pas couché comme 
un mort mais sur ses pieds - un autre rappel métaphorique de la vivacité de Molière. Puis la tombe se referme lentement, le «mort-vivant» qu'est le créateur/l'hérétique disparaît, et il ne reste plus qu'une dernière allusion à la scène blanche qui autrement resterait vide: la pierre tombale.

\section{NOTES}

1. "One can free-associate all these parts in multiple ways. There are no definite ways to link up the various images, sounds and other pieces of information. There are an infinite number of possibilities. It is a space in which we hear and see and experience, and then we make associations». Cf. «Robert Wilson and Umberto Eco : A

Conversation ", Performing Arts Journal 43, 1993, p. 89.

2. "[Wilson's plays'] structure is musical in the sense that the action is an architectural arrangement of sounds, words and movements, in which images are restated or varied to form thematic motifs». Cf. C. Innes, Avant Garde Theater, London \& New York, Routledge, 1993, p. 202.

3. C. G. Jung, The Psychology of Litterature, chap. 2 "The Artist", New York, Harcourt Brace, 1933.

4. M. Boulgakov, Le Roman de Monsieur de Molière, trad. du russe par M. Pétris, Paris, Gallimard, coll. "Folio», 1993, p. 189-190.

5. Boulgakov fait de La Grange le mémorialiste de la troupe depuis qu'il s'y est joint en 1659: il est le témoin qui enregistre les événements marquants de la vie de la troupe (ibid., p. 123-124).

6. En français dans le texte. Dorénavant tous les termes en italiques, sauf ceux qui sont dans une langue étrangère, indiqueront cette particularité du texte original (N.D.T.).
7. Boulgakov, op. cit., p. 14

8. J. Linders, "Molière \pm Müller", Performance Research, vol. 1, n², 1996, p.93.

9. H. Müller, Hamlet Machine, Paris, Éd. de Minuit, 1979.

10. «[...] as soon as montage intervenes [...] the present becomes the past [...]. It is thanks to death that our lives become expressive. Montage thus accomplishes for the material of film [...] what death accomplishes for live». Cf. P. P. Pasolini, "Observations on the Long Take», October 13 1990, p. 5-6 (traduction de J.-P. Vidal).

11. R. Barthes, La Chambre claire. Note sur la photographie (1980), dans Eurres Complètes, tome III, 1974-1980, Paris, Éd. du Seuil, 1995, p. 1118.

12. Boulgakov établit un lien direct entre Molière, la philosophie épicurienne et la nouvelle science de son époque en décrivant ses études avec Pierre Gassendi qui fut lui-même accusé d'hérésie. La Fontaine fut aussi de ses élèves (ibid., p.44-49).

13. Cf. J.-P. Sartre, L'Imaginaire: psychologie phénoménologique de l'imagination, Paris, Gallimard, 1940.

14. Cette séquence semble former une variation sur une anecdote racontée par Boulgakov et dans laquelle Molière et l'enfant Baron se rencontrent pour la première fois, ce dernier jouant en secret sur le clavecin "magique" présenté au roi (ibid., p. 217-220). 